\title{
The Development of MaccabiDent Quality Measures for Oral Health
}

\author{
Manor $\mathrm{L}^{1}$, Bernstein $\mathrm{Y}^{1}$, Tobias $\mathrm{G}^{2 *}$, Librus $\mathrm{S}^{1}$, Rosen $\mathbf{R}^{1}$, Findler $\mathbf{M}^{1}$ and \\ Mann J1 \\ ${ }^{1}$ MaccabiDent, Dental Health Services, Israel \\ ${ }^{2}$ Department of Community Dentistry, Hebrew University, Israel
}

*Corresponding author: Guy Tobias, Department of Community Dentistry, Hebrew University-Hadassah School of Dental Medicine, Jerusalem, Israel, Tel: 972527052333; Email: Guy.tobias@mail.huji.ac.il

\section{Research Article \\ Volume 5 Issue 4}

Received Date: November 17, 2020

Published Date: November 25, 2020

DOI: $10.23880 /$ oajds- 16000280

\section{Abstract}

Introduction: Success depends, at least in part, on the quality of the product, quality of service, and quality of infrastructure. Lack of quality measures in dentistry limits improvements in oral health care. This article focused on developing quality measures in dentistry.

Materials and Methods: Quality measures were developed and data from a three-year period were evaluated for MaccabiDent, a provider of dental services in Israel, treating 650,000 patients a year. The following 4 measurements were examined: The percentage of orthodontic patients who underwent a bi-annual dental examination with $\mathrm{x}$-rays and were treated by a hygienist; The percentage of children aged 3-12, in a B or C risk group that had a fluoride application twice yearly; Continuing Education for dental care providers; Implant success rate.

Results: $43.3 \%$ of patients had at least one dental examination with x-rays. $76.5 \%$ of the children had a fluoride application at least twice a year, with an average of 58.9\% in all clinics. $13.8 \%$ of care givers reported participation in Continuing Education courses. Success rate of implants was $99 \%$.

Discussion: Our findings, based on three years of data, will provide the foundation for the development of measures that will improve quality in all 53 dental clinics of the Health Dental Organization (HDO).

Conclusion: The most important outcome of this article is to show the importance of determining and measuring quality variables which might serve as a lead for a national index.

Keywords: Quality; Prevention; Fluoride; Oral Health

Abbreviations: HDO: Health Dental Organization; IOM: Institute of Medicine; CE: Continuing Education; DQA: Dental Quality Alliance.

\section{Introduction}

Success depends on the quality of the products, services and infrastructure [1]. Therefore, improving the quality of health services is a key element for success. In order to improve outcomes, various aspects that are the most relevant or important to a specific service are selected as measures and then examined. Reliable measures may enable various stakeholders, such as policymakers and dentists, to evaluate and improve the quality of care. Quality measures should address quality across the continuum of care [2]. The domains used to assess quality of health care providers are based on the Donabedian framework [3] and are classified as: Structure, process and outcome. 


\section{Open Access Journal of Dental Sciences}

\section{Structural Measures}

Structure includes the variables and factors in the facility where health care is delivered. For example, equipment, proportion of board-certified specialists versus general practitioners, use of computerized platforms (electronic medical records, prescriptions etc.) and the caregiver to patient ratio.

\section{Process Measures}

Processes are the actions performed by the provider in order to maintain or improve health, from diagnosis to treatment, including preventive care, and patient education. For example, the percentage of people receiving preventive services, the percentage of people with high blood cholesterol values who had their blood lipid levels tested and correspondingly treated. The majority of health care quality measures are process measures because, according to Donabedian, the process encompasses all aspects of healthcare delivery [4].

\section{Outcome Measures}

Outcomes include the impact of the health care service on the health status of patients. For example, patient satisfaction, changes in health status and issues, that affect health related quality of life, rate of surgical complications, readmissions etc.

Many analytic frameworks for quality assessment have been developed for the public and private sectors. One such framework includes six domains for health care quality and was published by the Institute of Medicine (IOM) in the Department of Health and Human Services, in the USA in 2001 [5] the six domains are:

a) Safe: Avoiding harm to patients from care intended to help them.

b) Effective: Providing services based on scientific knowledge to all who could benefit and refraining from providing services to those not likely to benefit (avoiding underuse and misuse, respectively).

c) Patient-centered: Providing care that is respectful of, and responsive to, individual patient preferences, needs and values and ensuring that patient values guide all clinical decisions.

d) Timely: Reducing waiting time and sometimes harmful delays for those who receive and provide care.

e) Efficient: Avoiding waste of equipment, supplies, ideas, and energy.

f) Equitable: Providing care that does not vary in quality because of personal characteristics such as gender, ethnicity, geographic location, and socioeconomic status.
The lack of quality measures in dentistry has been a barrier to quality improvements in oral health care [6]. A systemic review, published in the Journal of Community Dentistry and Oral Epidemiology in 2018 [7] aimed to provide an overview of existing quality measures in the field of oral health care. The range and variety of articles dealing with quality measurements emphasizes the great importance investigators, dental providers and administrative personnel, place on measuring dental care and, consequently, improving it. 24 publications were included in the systemic review, yielding 215 quality measures, mostly referring to treatment and preventive services. Specifically, 108 measures were process measures (over 50\%), 84 (39\%) were outcome measures, $71(33 \%)$ dealt with oral treatment or preventive services and the others concerned with oral disease outcome, aspects of access to care, and oral health costs. Few structure measures were identified, focusing on patient safety, organized aspects of oral health care or costs of oral health.

Among the articles included in the above mentioned review, Hussein, etal. published a study performed in Germany in 2017 [8] aiming to increase patient safety, through the rational use of systemic antibiotics and increasing the use of first line medications. In 2016 Bhardwaj, et al. [9] in the USA, reported a meaningful dental quality measure based on the percentage of children, aged 0-20 years, receiving a topical fluoride varnish application. A study from Finland, published by Mattila, et al. [10], dealt with measuring the quality of children's dental health care, from the oral health care records of 10-year-old children, using 5 outcome measures. In 2017 in the USA Neumann A, et al. [11], evaluated the feasibility and validity of the Finnish measures.

A recent article published in the International Dental Journal aimed at developing quality measures for adults. According to the authors Righolt, et al. developed the first set of oral health quality measures for adults. The study provides insights "regarding which aspects of quality of oral health care are relevant, appropriate and important to measure according to the key stakeholders in the Dutch oral health care field" [12].

The importance of quality measurements in health, and specifically in dental health, have recently become clearer. This article describes the development of quality measures in dentistry, in a large number of dental clinics, which are part of the national health system in Israel and describes the initial findings from the data collected.

\section{Methods}

Israel's health system includes private practices, hospitals, the military, universities and four health funds, and is obligated to provide high quality medical services 
to all citizens. The ability to determine quality depends on the standards of the measures. Hospitals in Israel joined a worldwide trend of improving quality measures based on understanding the importance of quality in all aspects of medical care. In 2010, Israel joined the OECD, which recommended, amongst other things, developing quality measures in hospitals. In 2012, a committee was appointed by the Ministry of Health to establish recommendations to improve quality of care in all fields of medicine. There were many challenges when this national plan started in 2013: the need for standardization of medical files, choosing the right quality measures, and the need to improve quality of care [9]. There seemed to be a lack of parameters that allowed comparisons between systems, and there were no common denominators for comparisons. An effort was also made to develop quality measures in dentistry, and MaccabiDent, a large provider of dental services in the country and a subsidiary of the Maccabi Health Fund, decided to invest in the development of these measures.

Israel has approximately 6000 active dentists, and over 1100 are employed within the MaccabiDent system, which has 53 clinics with 397 dental units. This dental provider treats 650,000 patients a year, performing 2.8 million procedures annually. Since MaccabiDent is fully computerized and digitized, development of quality measures was feasible, but comparisons with other dental providers in Israel, which are not computerized to the same degree, was difficult. MaccabiDent began collecting data and comparing outcomes in their own clinics in 2016, (when the MaccabiDent system became fully computerized).

In the lastyears a committee dealing with the development of these measures, came up with a set of measures dealing with the most important activities of the dental clinics. These tools were suggested, based also on existing literature and were introduced gradually and evaluated once in a while, in an effort to finally come up with a set of measures which could be used within the company and finally be accepted as a national index to be used in Israel and may be in other systems around the globe. Today, MaccabiDent uses 16 quality measurements covering various areas and subjects. It is impossible to discuss all these indicators; therefore, we decided to evaluate and discuss four which represent various types of measures.

The aim of comparing clinics within the organization using the quality measures is to trigger "competition" in order to improve outcomes. The 16 quality measures MaccabiDent developed and follow are:

a) Measuring oral hygiene-all patients defined as having poor oral hygiene and not treated by a hygienist, within three months. b) Measuring all diagnosed diabetic patients, who did not receive treatment by a hygienist, within three months of diagnosis.

c) Measuring mandatory and voluntary Continuing Education (CE) provided to the dentists.

d) Measuring the failure of implants, within 12 months of insertion.

e) Measuring the percentage of patients, diagnosed with periodontal disease and not treated by a periodontist, within 6 months of diagnosis.

f) Measuring the percentage of prosthodontic treatments, in which no failure of treatment was reported, within one year.

g) Measuring the percentage of children ages 3-18, with a full treatment plan completed within 3 months.

h) Measuring the percentage of children ages 3-18, who returned for a checkup to the clinic they were treated in during the last year.

i) Measuring the percentage of orthodontic patients, who underwent an annual dental examination with x-rays, and 6 monthly hygiene treatment, by a hygienist.

j) Measuring the percentage of patients, who did not file a complaint regarding the services they received, within the year evaluated.

k) Measuring the effectiveness of a clinic, according to the percentage of patients who completed a treatment plan within a specific period of time.

l) Measuring the percentage of children ages 3-12, in a B or $\mathrm{C}$ caries risk group that received a topical fluoride varnish application twice a year.

m) Measuring the percentage of team members in a specific clinic, who received all required vaccinations.

n) Measuring all team members in a specific clinic, who had the Hepatitis B vaccination, determined by antibody levels.

o) Measuring the percentage of all temporary crowns built on a post and a core, within a specific period of time.

p) Measuring the percentage of completed root canal treatments, that had completed a post and a core on the tooth within 4 weeks.

\section{Results}

Only four quality measures of the 16 available were chosen for a discussion allowing examination of the advantages and disadvantages of these specific indicators based on clinical procedures and continuing education (CE). These were chosen as representatives of various types of 
measures.

1. Measuring the percentage of orthodontic patients, who underwent an annual dental examination with x-rays, and 6 monthly hygiene treatment, by a hygienist. The specific clinic presented here is ranked sixth among all 53 clinics evaluated, with $43.3 \%$ of patients fulfilling the requirement of the measure. The average for all clinics was $28.6 \%$. Furthermore $40.3 \%$ of patients in this clinic had two sessions with the hygienist, while the mean for all clinics was $34.7 \%$. The rank of the clinic was determined by averaging the two parameters. The range was from $15.2 \%$ for the clinic that needed to improve to $70 \%$ for the best clinic. The aim was for all clinics to be at least as good as the mean percent.

2. Measuring the percentage of children ages 3-12, in a B or $\mathrm{C}$ caries risk group that received a topical fluoride varnish application twice a year. For this specific measure, 76.5\% of the children received the application at least twice a year, with an average of $58.9 \%$ in all clinics. The range for this quality measure was between $48.5 \%$ and $76.5 \%$. We also measured the percentage of children ages 3-12 who received a fluoride varnish application out of all children who visited MaccabiDent clinics during that month Figure 1. For this specific measure, we found that more than $50 \%$ of the children who visited the clinics each month received a fluoride application, with a range of $59.4 \%$ (September/19) to $54.2 \%$ (May/20). The decrease in the percentage of children receiving fluoride was due to the COVID -19 closure in which only some of the clinics provided care.

3. Continuing Education (CE) for dental care providers. This includes mandatory courses (defined by the ministry of Health) and participation in voluntary CE courses, based on a declaration by the care provider for 2019. In the specific clinic presented here, $20.1 \%$ participated in mandatory courses and $13.8 \%$ reported participation in other CE courses. The mean for all clinics was $12.5 \%$ for mandatory courses and $4.1 \%$ for other CE courses. In addition, 56.8\% reported partaking in CE courses for which an official document was presented. None of the clinics recorded $100 \%$ participation in any given year since the CE courses were measured over a three-year period. In 2019 the range was between $12.4 \%$ for the weakest clinic to $65.5 \%$ for the strongest. This wide range indicates serious discrepancies which need to be evaluated and corrected. The mean participation in all clinics for CE, was $34.2 \%$. The specific clinic presented was ranked as the best of all clinics evaluated Figure 2.

4. Measuring success rate of implants, one year after insertion. All clinics had a very low failure rate. The best clinic had $100 \%$ success rate, and the range was between 99-100\%. The success rate in most clinics evaluated was higher than 99\%. The very high success rate, in all clinics, indicates that this specific measure should not be part of a set of measurements of quality. Figure 3 compares the weighted evaluation of all 16 measures starting in 2016 with 39.7\%, 2017, 43.1\%, $201855.0 \%$ and 60.9\% in 2019. This tool not only allowed a comparison between clinics, but the accomplishments over the years could be monitored. Figure 4, describes the mean weighted quality measurements in relation to socio-economic clusters. No differences were noticed between the various clinics around the country.

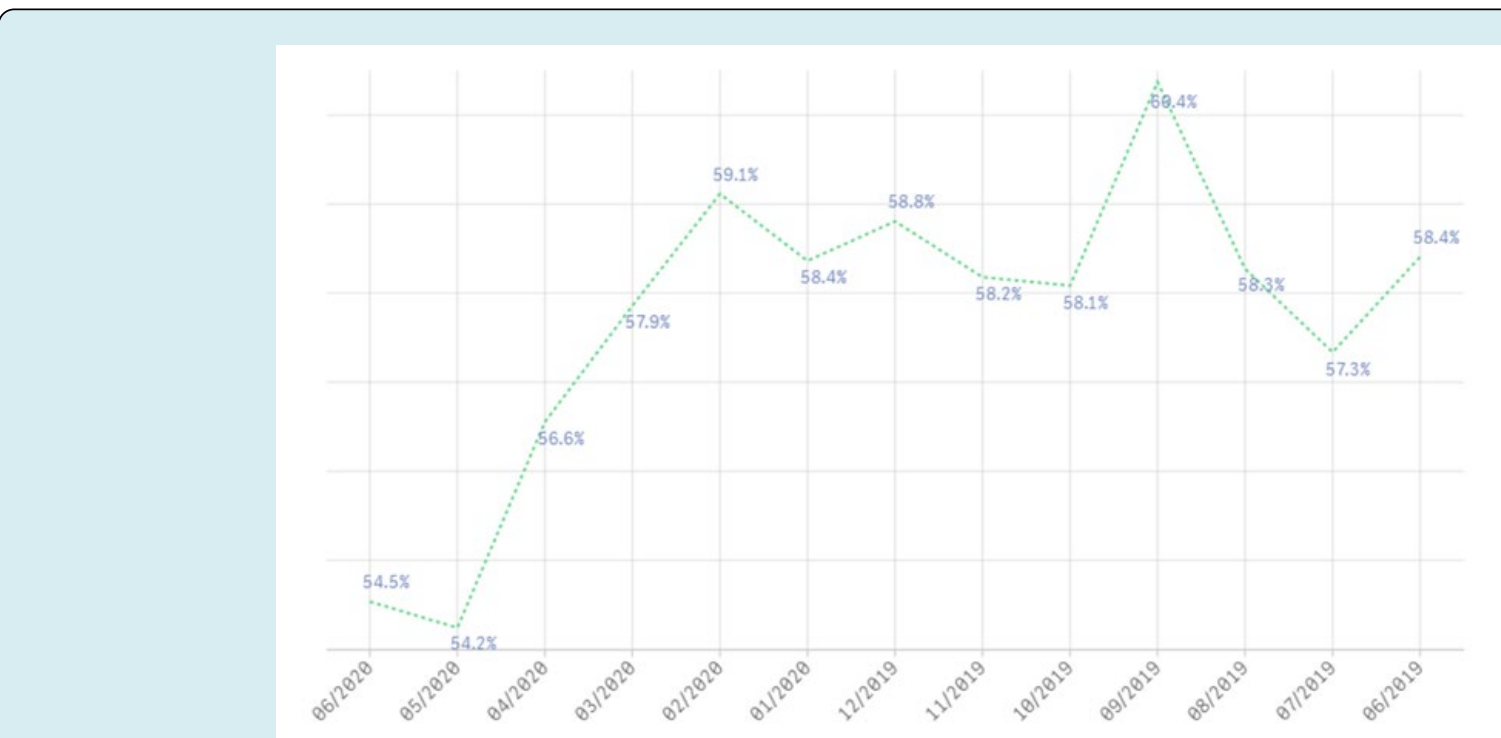

Figure 1: Rate of children ages 3-12 who received fluoride application out of all children visiting MaccabiDent clinics. 


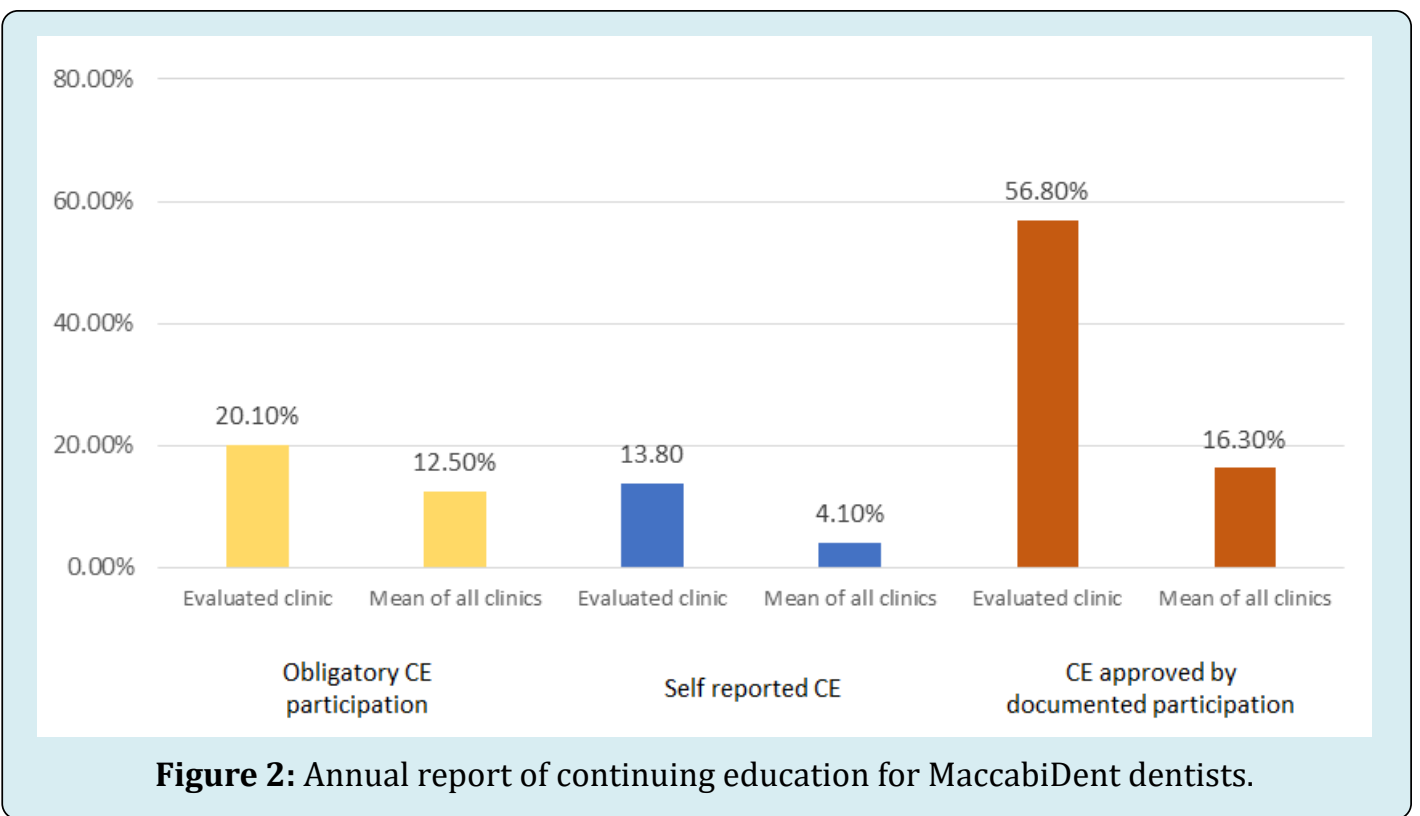

Figure 2: Annual report of continuing education for MaccabiDent dentists.
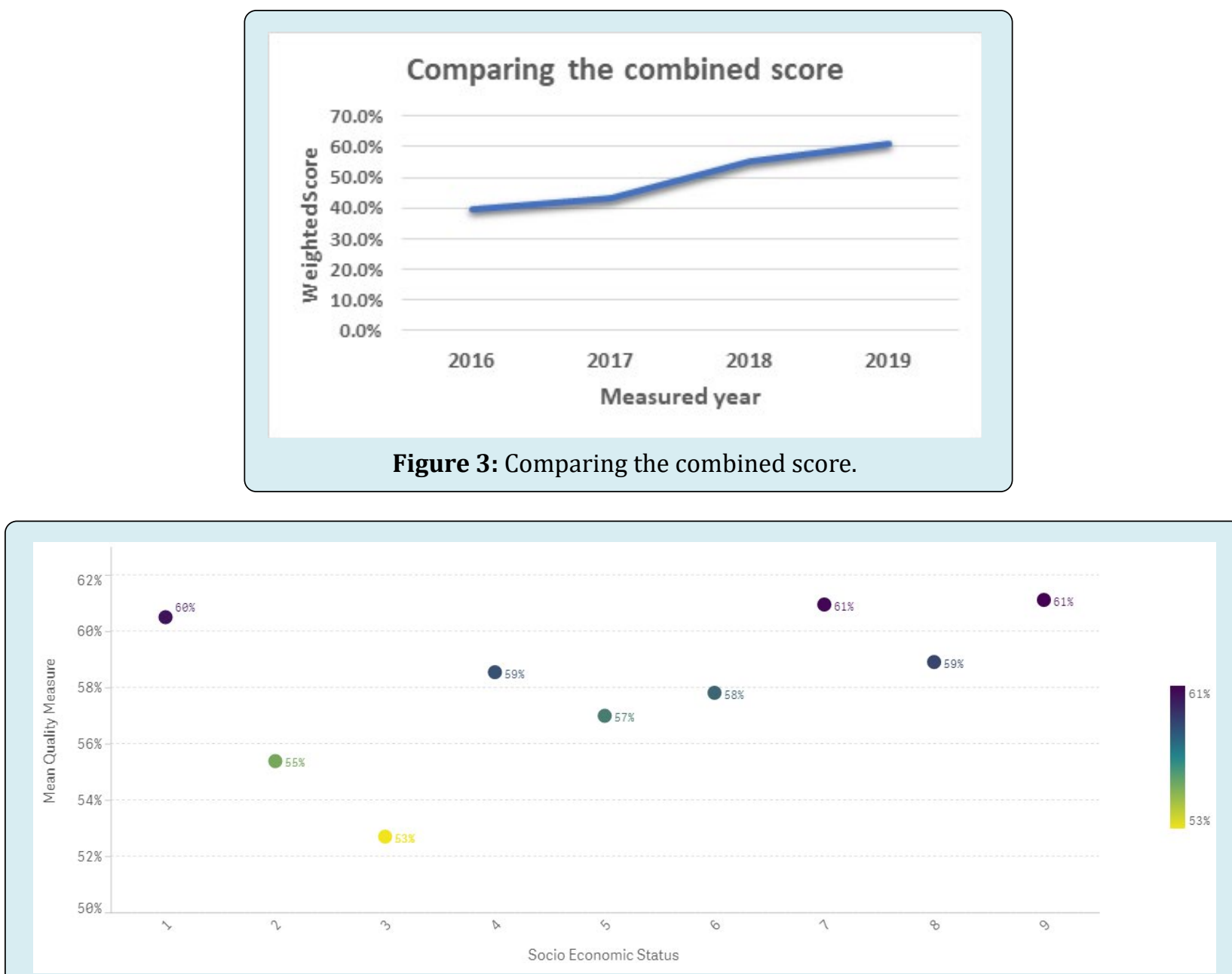

Figure 4: Mean quality measures as related to Socio-Economic status based on clinic location. 


\section{Open Access Journal of Dental Sciences}

\section{Discussion}

The mission of the Dental Quality Alliance (DQA) [13] was to "Advance the field of performance measurement to improve oral health, patient care, and safety, through a consensus building process." The first objective of the DQA was to "identify and develop evidence-based oral health care performance and measurement resources". In June 2016 the American Dental Association, on behalf of the DQA, published the guide book "Quality measures in Dentistry" [14], which discusses many aspects of quality measures and emphasizes the importance of these measurements. According to the guide, the measures show where systems are breaking down and where they are successful and enable care providers to help patients get and stay well. The measures facilitate selfreview of performance by healthcare providers and highlight how improvements in measures are due to improved care. All measures aim to advance healthcare quality. Today, all individuals involved in providing care, including: administrators, care givers and stakeholders, would like to see a higher standard of care, which is measurable and allows, not only improved patient care, but also acts as a tool to evaluate cost and benefits.

Will a successful facility provide better care? Will continuing education improve care? Will having more administrators improve care? Will new equipment, such as scanners, improve care? These and many other measures are important to the desired outcome, i.e. improved care. To the best of our knowledge, no standardized set of measures exists, and it is clear that in order to be able to compare providers - private, public, governmental, military and others, we need a set of measurements, that are universally accepted and adopted, as has been achieved in other areas, such as a standard for accreditation in dental schools around the world.

There seems to be a consensus, within the profession, that the quality of measures is important, yet, we are far away from defining which measures to employ. MaccabiDent, which is a large provider, supported by a Health Care Organization via the Ministry of health in Israel, is creating the first set of measures to be used, hopefully, in all clinics in Israel. The quality measurements developed in MaccabiDent aimed to cover all aspects of dental care.

On the one hand, the measurement dealing with failure of implants within the first year of insertion, showed an extremely low failure rate of around $1 \%$, for all clinics. This uniformly high success rate indicates that such a measure is not useful to improving outcomes (which are essentially already perfect) and therefore this measure would likely not be included. On the other hand, the measure of orthodontic examination, $\mathrm{x}$-rays once a year and meeting a hygienist twice a year, ranged between $16 \%$ and $67 \%$ with a mean of $28.8 \%$ for all 53 clinics in the chain. The large variation emphasizes that the clinics ranked low on the 53-clinic scale, can and should improve, at least up to the mean rate for all clinics. Application of fluoride is another significant measure, because of the variation in results. Continuing education measured by both participating in compulsory courses and by other means of education, is important for keeping up to date and presumably improving quality of care.

We believe that the mostimportant outcome of this article would be to advance the understanding of the importance of measuring variables in improving quality. Different measures could obviously be used instead of, or as well as, the ones we chose and discussed. Measures are important, as they enable evaluation of the quality of care within each clinic of MaccabiDent. The results facilitate comparison between the clinics and motivate the team members to improve. The data collected for quality measures could also be used in research, such as the effect of fluoride application on caries at different ages.

Due to the fact that dental clinics are influenced by many factors, quality measures need to be updated and improved. Each measure should consider the quality of care, while understanding long term costs and benefits. It may be more practical to divide the measures into categories e.g. those evaluating clinical procedures, outcomes of CE for dentists, hygienists, and dental assistants, quality of infrastructure and its effect on quality and satisfaction of care by patients.

We discovered that the more we investigated this issue, with hundreds of published papers in the literature, the more complicated we found it. Hence, we decided to initially concentrate on the clinical aspects and then develop other sets of measures dealing with infrastructure and its effect on clinical quality, and the importance of CE and its effect on quality of care. Furthermore, it may be impossible to find universal quality measures, as they might vary, according to country, culture, financial basis and other factors.

MaccabiDent decided to continue to study and develop these measures, and then make the scale available to all clinics in Israel and maybe other interested countries.

\section{Conclusion}

In conclusion, quality measures are important in every discipline, especially, in medicine and dentistry. The Israeli Ministry of Health initiated a national program for quality indicators, for general and geriatric Hospitals, psychiatric hospitals, mother and child health centers and medical services [15]. These measures were implemented in 2013. MaccabiDent decided to develop a set of measures for the 
improvement of quality and service. This article presents the interim results, following three years of development and measurements. These findings will serve as a base for improving quality in all dental clinics i.e. those supported by the government and private clinics. The set of measures, once fully employed, will allow evaluation of every clinic and find areas that can be improved, for the benefit of patients and care givers.

\section{References}

1. Campbell SM, Kontopantelis E, Hannon K, Burke M, Barber A, et al. (2011) Framework and indicator testing protocol for developing and piloting quality indicators for the UK quality and outcomes framework. BMC Fam Prac 12: 85.

2. Adirim T, Meade K, Mistry K (2017) A New Era in Quality Measurement: The Development and Application of Quality Measures. Pediatrics 139(1): e20163442.

3. Ayanian JZ, Markel H (2016) Donabedian's Lasting Framework for Health Care Quality. N Engl J Med 375(3): 205-207.

4. Donabedian (2003) An introduction to quality assurance in health care. $1^{\text {st }}$ (Edn.), New York, NY: Oxford University Press.

5. Institute of Medicine (IOM) (2001) Crossing the Quality Chasm: A New Health System for the 21st Century. Washington, D.C: National Academy Press.

6. Institute of Medicine (IOM) (2011) Committee on an oral health initiative. Advancing Oral Health in America. Washington, DC: National Academics Press.
7. Righolt AJ, Sidorenkov G, Faggion CM, Listl S, Duijster D (2019) Quality measures for dental care: A systematic review. Community Dent Oral Epidemiol 47(1): 12-33.

8. Hussein RJ, Krohn R, Kolle PK, Willms G (2017) Quality indicators for the use of systemic antibiotics in dentistry. Z Evid Fortbild Qual Gesundhwes 122: 1-8.

9. Bhardwaj A, Ramoni R, Kalenderian E, Neumann A, Hebbali NB, et al. (2016) Measuring up: Implementing a dental quality measure in the electronic health record context. J Am Dent Assoc 147(1): 35-40.

10. Mattila ML, Rautava P, Paunio P, Ojanlatva A, Hysalla L, et al. (2002) Children's dental healthcare quality using several outcome measures. Acta Odontol Scand 60(2): 113-116.

11. Neumann A, Kalenderian E, Ramoni R, Yansane A, Tokede B, et al. (2017) Evaluating quality of dental care among patients with diabetes: adaptation and testing of a dental quality measure in electronic health records. J Am Dent Assoc 148(9): 634-643.

12. Righolt AJ, Duijster D, Van der Wees PJ, Listl S, Smits KPJ (2020) Dutch oral health care quality measures: a modified Delphi study. Int Dent J 70(4): 277-286.

13. Aravamudhan K Dental Quality Alliance Measures.

14. (2016) Quality Measurement in Dentistry, A Guidebook. Dental Quality Alliance.

15. National Program for Quality Indicators. Israel Ministry of Health.

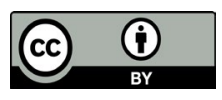

